\title{
Partial Deconfinement
}

\author{
Masanori Hanada, ${ }^{a}$ Goro Ishiki ${ }^{b, c}$ and Hiromasa Watanabe ${ }^{c}$ \\ ${ }^{a}$ School of Physics and Astronomy, and STAG Research Centre, University of Southampton, \\ Southampton, SO17 1BJ, U.K. \\ ${ }^{b}$ Tomonaga Center for the History of the Universe, University of Tsukuba, \\ Tsukuba, Ibaraki 305-8571, Japan \\ ${ }^{c}$ Graduate School of Pure and Applied Sciences, University of Tsukuba, \\ Tsukuba, Ibaraki 305-8571, Japan \\ E-mail: m.hanada@soton.ac.uk, ishiki@het.ph.tsukuba.ac.jp, \\ watanabe@het.ph.tsukuba.ac.jp
}

ABSTRACT: We argue that the confined and deconfined phases in gauge theories are connected by a partially deconfined phase (i.e. $\mathrm{SU}(M)$ in $\mathrm{SU}(N)$, where $M<N$, is deconfined), which can be stable or unstable depending on the details of the theory. When this phase is unstable, it is the gauge theory counterpart of the small black hole phase in the dual string theory. Partial deconfinement is closely related to the Gross-Witten-Wadia transition, and is likely to be relevant to the QCD phase transition.

The mechanism of partial deconfinement is related to a generic property of a class of systems. As an instructive example, we demonstrate the similarity between the Yang-Mills theory/string theory and a mathematical model of the collective behavior of ants [Beekman et al., Proceedings of the National Academy of Sciences, 2001]. By identifying the D-brane, open string and black hole with the ant, pheromone and ant trail, the dynamics of two systems closely resemble with each other, and qualitatively the same phase structures are obtained.

KEYWORDS: Black Holes in String Theory, Gauge-gravity correspondence

ArXIV EPRINT: 1812.05494 


\section{Contents}

1 Introduction 1

2 Partial deconfinement 4

3 Intuition from black hole/ant trail correspondence $\quad 5$

4 Quantitative tests with Polyakov line phases $\quad 9$

$\begin{array}{ll}4.14 \mathrm{~d} \mathcal{N}=4 \mathrm{SYM} \text { on } \mathrm{S}^{3} & 10\end{array}$

4.2 Other $4 \mathrm{~d}$ theories on $\mathrm{S}^{3} \quad 11$

$4.34 \mathrm{~d}$ pure Yang-Mills on flat space 11

4.4 Matrix quantum mechanics 11

4.5 2d maximal SYM and black hole/black string topology change 13

$\begin{array}{lll}5 & \text { Conclusion and discussions } & 14\end{array}$

$\begin{array}{ll}\text { A Small black hole from gauge theory } & 17\end{array}$

$\begin{array}{lr}\text { B The large } N \text { limit of the ant equation } & 18\end{array}$

B.1 Modified ant model (Ant-Man model) 20

C Monte Carlo simulation of the matrix model 20

\section{Introduction}

A longstanding problem in quantum field theory is understanding the details of the finite temperature deconfinement transition in QCD, and gauge theories more broadly. For QCD, this transition can be probed experimentally in heavy ion collisions, and can play an important role in the physics of the early universe. A central difficulty in theoretically studying the transition is the presence of strong interactions, rendering perturbation theory futile and requiring numerical techniques.

Certain strongly coupled gauge theories can be described by weakly coupled gravity via holographic duality [1-3]. According to the duality, the deconfinement transition in the gauge theory is equivalent to the formation of a black hole. The duality allows us to learn about the nature of the deconfinement transition from the perspective of gravity, and at the same time, the microscopic quantum gravitational aspects of the formation and evaporation of black hole are encoded in gauge theory.

The most well-studied example of holographic duality is the one between $4 \mathrm{~d} \mathcal{N}=$ 4 super Yang-Mills on the three sphere and type IIB superstring theory on $\operatorname{AdS}_{5} \times \mathrm{S}^{5}$ spacetime $[3,4]$. One can infer and study the microcanonical phase structure of the strongly 

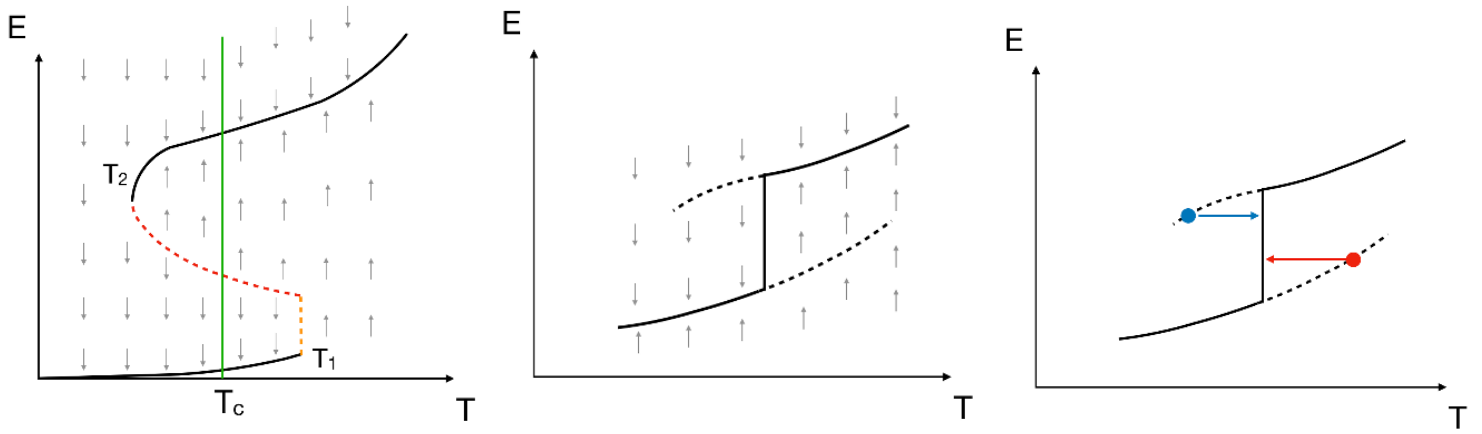

Figure 1. [Left] The phase structure of $4 \mathrm{D} \mathcal{N}=4 \mathrm{SYM}$ on $\mathrm{S}^{3}$. The orange and red dashed lines denote the Hagedorn string phase and small black hole phase, respectively, which are well-defined in the microcanonical theory. In the canonical treatment, the small black hole phase is the unstable saddle which is responsible for strong hysteresis. The green line marks the transition temperature in the canonical ensemble, $T_{c}$. Above $T_{c}$ the large black hole phase is favored, while below $T_{c}$ the graviton gas phase is favored. [Center] A more common example of a first order transition, for example, between ice and liquid water. Small perturbations can destabilize the metastable states. At the critical temperature, there can be a mixture of two phases, in which ice and liquid water coexist. [Right] Superheated ice (red point) and supercooled liquid water (blue point) are not stable even in the microcanonical ensemble. There is an instability toward the mixture of the ice and liquid water.

coupled region of super Yang-Mills from the weakly coupled gravity dual, which is depicted in the left panel of figure 1. There are a large black hole phase (black solid line at large energy region), a small black hole phase (red dashed line), a Hagedorn string phase (orange dashed line), and a graviton gas phase (black solid line at low energy region) $[4,5]$.

The large black hole has a positive specific heat, i.e. energy increases with temperature, as $E \sim N^{2} T^{4}$. On the other hand, the small black hole has a negative specific heat. When the black hole is much smaller than the curvature scale of $\operatorname{AdS}_{5} \times \mathrm{S}^{5}$, it is approximately the same as the Schwarzschild black hole in ten spacetime dimensions, and $E \sim N^{2} T^{-7}$. This small black hole phase is interesting, in part because it provides a microscopic description of black holes with negative specific heat, which is a proxy for evaporating black holes. In terms of the canonical ensemble, the small black hole phase is described by an unstable saddle, in the following sense. Let us write the canonical partition function at temperature $T$ as

$$
Z(T)=\int d E \Omega(E) e^{-E / T}=\int d E e^{-F(E, T) / T},
$$

where $\Omega(E)=e^{S(E)}$ is the density of states, $S(E)$ is the microcanonical entropy, and $F(E, T)=E-T S$ is the free energy. The saddles of $F$ for fixed $T$ satisfy

$$
0=\frac{\partial F}{\partial E}=1-T \frac{d S}{d E}
$$

where $(d S / d E)^{-1}$ is, by definition, the temperature in the microcanonical ensemble. Hence, the value of the energy $E(T)$ at the saddle, as a function of the temperature $T$, is the energy in the microcanonical ensemble. The stable saddles (local minima) correspond 
to the graviton gas and large black hole, whereas the unstable saddle (local maximum) corresponds to the small black hole. In the canonical ensemble (i.e. for fixed $T$ ), if we drive the system away from the minimum, it rolls back to the same minimum. The direction of the change, which is determined by the sign of $d S / d E$, is shown by gray arrows in the left panel of figure 1 above. In other words, the metastable phases (i.e. the large black hole phase at $T<T_{c}$ and the graviton gas phase at $T>T_{c}$ ) are stable against small perturbations. This stability is unlike many standard examples of first order phase transitions, such as the transition between ice and liquid water. For example, we can supercool liquid water below its freezing temperature, but ice appears as soon as a tiny perturbation is added. In the case of ice and liquid water, as shown in the center panel of figure 1, the mixture of two phases can exist at $T=T_{c}$ (the solid vertical line). The direction toward equilibration is shown by gray arrows, and there is no unstable saddle. ${ }^{1}$ Even in the microcanonical ensemble, as shown in the right panel of the figure, the supercooled and superheated phases are unstable towards the mixture of ice and liquid water. ${ }^{2}$ Such instability is absent in the microcanonical treatment of the black hole and super-Yang-Mills.

From the gravitational point of view, the phase structure in the left-panel of figure 1 is easy to understand $[4,5]$. But by holographic duality, there is necessarily an alternative description of the phase structure in terms of the dual gauge theory. While various features of the phase structure have familiar counterparts in gauge theories, the counterpart to the small black hole phase is unfamiliar, and begs a gauge theory description. Previously, it has been proposed [6] that the mechanism which we call partial deconfinement can naturally explain the gauge theory counterpart to the small black hole phase. Berenstein [7] used a simple matrix model and combinatorial arguments to justify partial deconfinement. His arguments suggest that partial deconfinement is a generic feature of gauge theories, even without a gravity dual. In this paper, we leverage gauge theory calculations and numerics to provide compelling evidence in support of partial deconfinement, and give an intuitive explanation of the mechanism behind it. In particular, we will see that the distribution of Polyakov line phases contains rich information about the partially deconfined phase. Our results suggest that partial deconfinement is universal across a broad class of supersymmetric and non-supersymmetric gauge theories, and approximately applies to real-world QCD.

This paper is organized as follows. In section 2, we introduce the notion of partial deconfinement. In section 3, we show that the partial deconfinement phase in Yang-Mills is well-captured by models of collective motion with positive feedback. For concreteness, we consider a well-known model of ant trail formation. Intuition from the ant model leads

\footnotetext{
${ }^{1}$ More precisely, although the unstable saddle could exist very close to the 'metastable' state, a tiny perturbation which does not depend on the volume of the system is sufficient to escape from the metastable state by going beyond the unstable saddle. In Yang-Mills, the size of the perturbation necessary for going beyond the unstable saddle increases with $N$. In this sense, the 'metastable' phase in Yang-Mills is actually 'stable' in the large- $N$ limit.

${ }^{2}$ In the high energy theory/string theory community, the phrase 'two-phase coexistence' is sometimes used sloppily, and has two different meanings: (i) the mixture of two phases, such as liquid and solid phases, and (ii) existence of two (meta-)stable phases at the same temperature. In order to avoid the confusion, we will not use this terminology.
} 
us to a unified perspective on the phase structures of various Yang-Mills theories with and without first order transitions. In section 4 , we provide quantitative analytic and numerical evidence of the partial deconfinement phase in various models. In section 5 , we conclude with a discussion, including potential application to QCD. We collect various details of our calculations and numerical simulations in the appendices.

\section{Partial deconfinement}

As mentioned in the introduction, $4 \mathrm{~d} \mathcal{N}=4 \mathrm{SYM}$ has a peculiar phase structure. Its peculiarities can be understood by examining the kinds of degrees of freedom which characterize various phases. In the water/ice example two phases can occupy different regions in space at the freezing temperature, and such mixture of two phases connects the completely-liquid and completely-solid phases. We can supercool or superheat the system, but however large the volume of the full system, a perturbation localized in space can create a bubble of a more stable state, which then spreads to fill the entire volume. In the $4 \mathrm{~d} \mathcal{N}=4 \mathrm{SYM}$, it is natural to assume two phases (confined and deconfined) can occupy different regions in color degrees of freedom [6-8], namely $\mathrm{SU}(M)$ in $\mathrm{SU}(N)$, where $M<N$, is deconfined. Let us call it partial deconfinement, as coined in ref. [7].

In the language of D-branes and open strings, $M$ of $N$ D-branes are connected by open strings and form a bound state (black hole). It can be regarded as 'the mixture of two phases' from the D-brane point of view. The difference from the example of water is that the locations of D-branes is described by the internal degrees of freedom (color degrees of freedom) in QFT, rather than the spatial coordinate. Note that the interaction between D-branes can be highly nonlocal, in that all D-branes in the bound state interact with each other via open strings. This is in a stark contrast with the case of water.

In some cases, partial deconfinement can lead to a negative specific heat because the number of unlocked degrees of freedom changes [6,9]. In the partially deconfined phase, the number of unlocked degrees of freedom participating in the dynamics is proportional to $M^{2}$, rather than $N^{2}$. (In terms of string theory, $M$ D-branes are forming a bound state, and open strings between them are excited.) When the energy $E$ is increased, more strings can be excited, and hence $M$ increases with $E$. But then the energy per degree of freedom ( $\simeq$ temperature), which is proportional to $E / M^{2}$, can increase or decrease, depending on the details of how $M$ depends on $E$. Thus negative specific heat can arise [9, 10]. This is in sharp contrast to the completely deconfined phase, in which the number of degrees freedom is fixed and hence the specific heat has to be positive.

Our general arguments so far do not establish exactly what theories have the negative specific heat. In ref. [6], the properties of $4 \mathrm{~d} \mathcal{N}=4 \mathrm{SYM}$ has been used to explain $E / N^{2} \sim T^{-7}$ at low energies, which agrees with the equation of state of the tendimensional Schwarzschild black hole and hence is consistent with the AdS/CFT duality (see appendix A).

As another set of examples, large- $N$ pure Yang-Mills in $3 \mathrm{~d}$ and $4 \mathrm{~d}$ flat space with large volume are known to have first order deconfinement transitions. Numerical simulations demonstrate the existence of strong hysteresis which resembles the left panel of 
figure $1[11,12]$. This can also be understood as a phase separation in the color degrees of freedom due to the unstable saddle (partial deconfinement): although spatially localized small bubble of a more stable state (the confined phase surrounded by the completely deconfined phase, or vice versa) can spread once it is created, the creation of the bubble itself can be suppressed at large $N$. The natural scale of the bubble is $1 / \Lambda_{\mathrm{QCD}}$, which is $N$-independent, and hence the size of the perturbation needed for the creation of the bubble increases with $N$. (Note that we are considering the 't Hooft large- $N$ limit, and hence large $N$ is taken before large volume. If the large volume is taken first, then a large enough fluctuation which goes beyond the partially deconfined phase would be generated somewhere. Note also that the same suppression can work for the spinodal decomposition as well.) Hence, as long as we start with the confined or completely deconfined phase and dial the temperature, we expect strong hysteresis.

Note that the partial deconfinement requires $N \geq 3$. Therefore, the $\mathrm{SU}(2) \mathrm{YM}$ should not have the first order transition - and it is actually the case, as demonstrated by lattice Monte Calro simulations.

In our language, the small black hole and Hagedorn string (the red and orange dashed lines in the left panel of figure 1) are partially deconfined. Partial deconfinement implies that the distribution of the Polyakov line phase should behave as ${ }^{3}$

$$
\rho(\theta)=\frac{N-M}{N} \rho_{\text {confine }}(\theta)+\frac{M}{N} \rho_{\text {deconfine }}(\theta)=\frac{N-M}{N} \cdot \frac{1}{2 \pi}+\frac{M}{N} \rho_{\text {deconfine }}(\theta),
$$

where $\rho_{\text {deconfine }}(\theta)$ is the distribution at the endpoint of the fully deconfined phase, $T=T_{2}$. At least in the examples we study in this paper, the completely deconfined phase is gapped (i. e. $\rho(\theta)=0$ at $\theta= \pm \pi$ ), and the partially deconfined phase is the non-uniform un-gapped phase. We conjecture that this is true in general.

In the past it has been argued that the gapped phase in $4 \mathrm{~d} \mathcal{N}=4 \mathrm{SYM}$ should be dual to the large black hole with positive specific heat $[5,13]$. It is consistent with our observation, because we are relating the partially deconfined phase to the negative specific heat.

\section{Intuition from black hole/ant trail correspondence}

The first order transition with an unstable saddle can be made transparent by considering a similar phase transition in the science of complex systems: the formation of the ant trail. This is one of the basic problems in the science of the collective animal behavior, and a concrete mathematical model is given and tested experimentally [14]. More broadly, this is a common feature of systems with collective motion and positive feedback. A crucial feature is that when many ants follow the same trail, the strength with which other ants are drawn to follow to trail is enhanced. Likewise, D-branes forming a large bound state have enhanced interactions which D-branes not in the bound state. By identifying ants and D-branes in an appropriate manner, the formation of the ant trail can be identified

\footnotetext{
${ }^{3}$ We will show the heuristic derivation in section 4 .
} 
with the formation of a black hole, and remarkably we can reproduce the essential features of the black hole.

Consider a colony of $N$ ants, where we take $N$ to be parametrically large. To simplify matters, we assume there is only one source of food, which is concentrated at some particular location. As ants walk away from the nest, some will find the food source and bring pieces back to the nest, leaving a pheromone trail along the way to direct other ants to the food source. Let $N_{\text {trail }}$ be the number of ants rallying on a single trail between the nest and the food source. The strength with which ants not on the trail are drawn to the trail is proportional to the amount of secreted pheromones $p N_{\text {trail }}$, where $p$ is the pheromone contribution from each ant on the trail. Hence, the interaction between ants not on the trail, and the pheromones along the trail, is enhanced as $N_{\text {trail }}$ becomes larger. This is similar to the case of gauge theory: if $N_{\mathrm{BH}}$ D-branes form a bound state and one of the remaining $N-N_{\mathrm{BH}}$ D-branes comes close by, ${ }^{4}$ there are $N_{\mathrm{BH}}$ different open strings which try to capture that D-brane. ${ }^{5,6}$ At higher temperatures, each open string mode can be more highly excited and thus can contribute more to the dynamics. Therefore, higher temperatures correspond to larger values of the pheromone parameter. Hence the basic correspondence is

$$
\begin{aligned}
N_{\text {trail }} & \longleftrightarrow N_{\mathrm{BH}}, \\
p & \longleftrightarrow T .
\end{aligned}
$$

The phenomenological equation introduced in ref. [14] is ${ }^{7}$

$$
\begin{aligned}
\frac{d N_{\text {trail }}}{d t} & =(\text { ants coming into the trail })-(\text { ants leaving the trail }) \\
& =\left(\alpha+p N_{\text {trail }}\right)\left(N-N_{\text {trail }}\right)-\frac{s N_{\text {trail }}}{s+N_{\text {trail }}} .
\end{aligned}
$$

Here $\alpha$ describes the probability that each ant accidentally find the food source, and $s$ determines the rate that ants leave the trail. The parameters $p, \alpha$ and $s$ depend on the species, geography around the colony, weather and so on. The size of the stationary ant trail can be obtained by solving the equation $\frac{d N_{\text {trail }}}{d t}=0$.

In the original treatment [14], $p, \alpha$ and $s$ are fixed and $N_{\text {trail }}$ has been calculated as the function of $N$. For our purposes, in order to make contact with black holes and gauge theory, we fix $\alpha, s$ and $N$, and calculate $N_{\text {trail }}$ as the function of $p$. As discussed in appendix B, the natural large- $N$ limit of the ant model is $\alpha \sim N^{0}, p \sim N^{0}, s \sim N^{1}$. In figure 2, we show the plot of $x \equiv \frac{N_{\text {trail }}}{N}$ versus $p$ for $\alpha=1, \tilde{s} \equiv \frac{s}{N}=0.1,1.0,5.0$, and $N=10^{5}$. (See appendix B for the analytic argument and 'physical' intuition.) The saddles (the solutions of $\frac{d N_{\text {trail }}}{d t}=0$ ) appear when the inflow and outflow of the ants (the first and

\footnotetext{
${ }^{4}$ Below we use $N_{\mathrm{BH}}$ instead of $M$, because it is identified with the number of D-branes forming the black hole.

${ }^{5}$ This mechanism is also known as the moduli trapping [15] and is applied to the inflationary cosmology.

${ }^{6}$ This can also be interpreted as the entropic force associated with the enhancement of the degrees of freedom from $N_{\mathrm{BH}}^{2}$ to $\left(N_{\mathrm{BH}}+1\right)^{2}$. The entropic force exists for any gauge theory, regardless of the existence of the dual gravity description.

${ }^{7}$ In the original notation in ref. [14], $N_{\text {trail }}$ and $p$ are $x$ and $\beta$, respectively.
} 

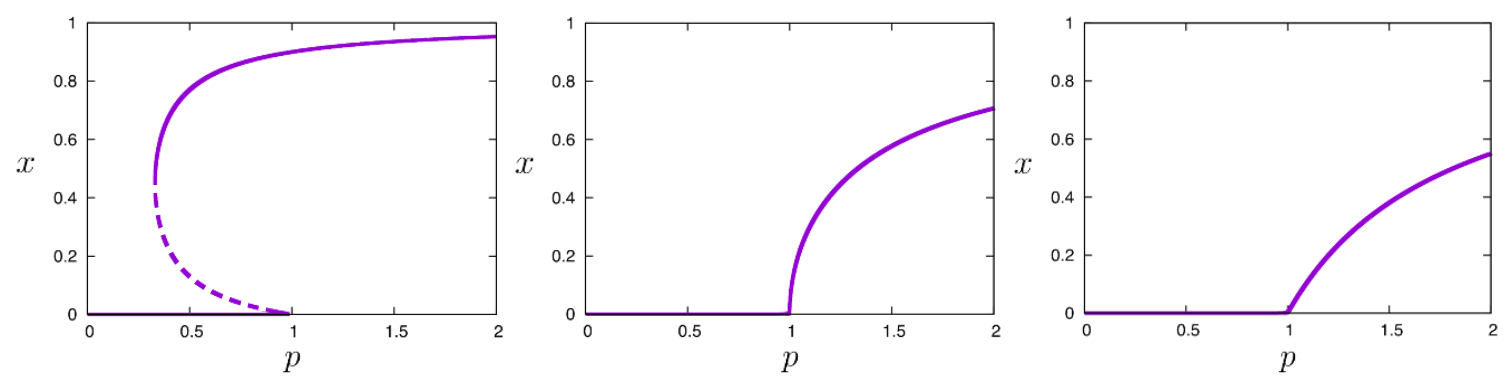

Figure 2. $x \equiv \frac{N_{\text {trail }}}{N}$ versus $p$ in the ant trail model (3.3). $\alpha=1, \tilde{s} \equiv \frac{s}{N}=0.1$ (left), 1.0 (center) and 5.0 (right), $N=10^{5}$.

second terms of (3.3)) balance. There are two cases, with and without the unstable saddle (dashed line):

- If the inflow/outflow wins when $N_{\text {trail }}$ is varied slightly upwards/downwards from the saddle, the saddle is unstable (dashed line in the left panel of figure 2). It happens at $\tilde{s}<1$.

- If the inflow/outflow wins when $N_{\text {trail }}$ is varied slightly downwards/upwards from the saddle, the saddle is stable (solid line). At $\tilde{s}>1$, only the stable saddle exists.

- The boundary of these two behaviors can be seen at $\tilde{s}=1$ (the center panel of figure 2). The curve $x=x(p)$ has infinite slope at $p=1$ in the large- $N$ limit.

The crucial feature responsible for the emergence of the unstable saddle is the positive feedback: near the unstable saddle, if $N_{\text {trail }}$ increases/decreases a little bit, then more ants join/leave the trail and $N_{\text {trail }}$ increases/decreases even more. The value of $N_{\text {trail }}$ at the unstable saddle decreases as $p$ is increased - negative specific heat, by interpreting larger $N_{\text {trail }}$ corresponds to larger energy - because with larger value of $p$ strong enough total pheromone can be obtained with smaller value of $N_{\text {trail }}$.

Essentially the same mechanism exists in gauge theory. In terms of strings and Dbranes: as $N_{\mathrm{BH}}$ grows, external D-branes are attracted more strongly (inflow); on the other hand, as $N_{\mathrm{BH}}$ grows, it costs more energy, and hence the Boltzmann factor pushes $N_{\mathrm{BH}}$ down (outflow); the saddle appears when these two effects balance. Again, whether the saddle is stable or unstable depends on the details of the dynamics. In figure 3 and figure 4, we show three possibilities:

- If the inflow/outflow wins when $N_{\mathrm{BH}}$ is varied slightly upwards/downwards from the saddle, the saddle is unstable (the dashed line in the left panels of figure 3 and figure 4). In order for this to happen, the strings have to bind D-branes tighter, so the strong coupling dynamics is needed. Indeed, this is the case for the strongly coupled region of $4 \mathrm{~d} \mathcal{N}=4 \mathrm{SYM}$.

- If the inflow/outflow always balances, no particular value of $N_{B H}$ is favored (the vertical solid line in the center panels of figure 3 and figure 4 ). This is the case for the weakly coupled region of $4 \mathrm{~d} \mathcal{N}=4 \mathrm{SYM}$. 

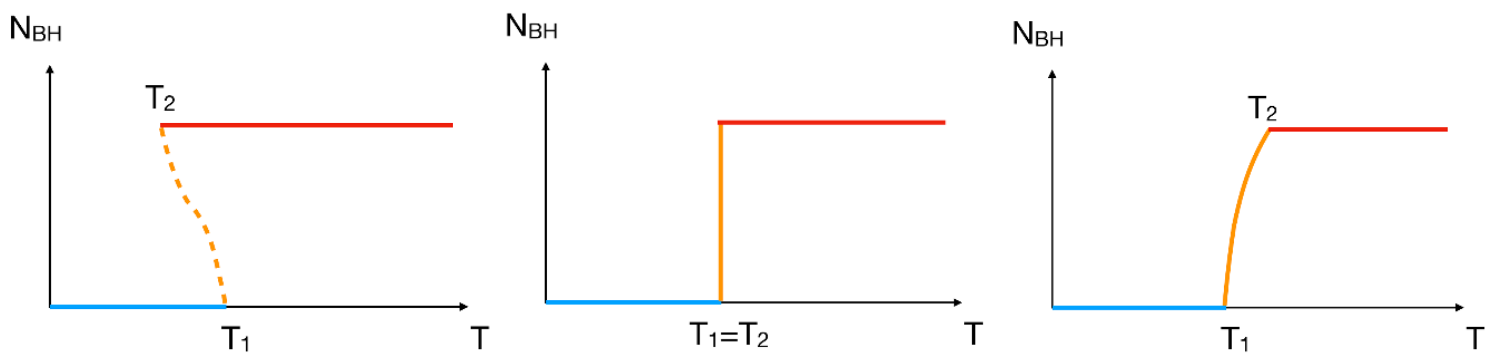

Figure 3. Cartoon pictures of $T$ vs $N_{\mathrm{BH}}$ in gauge theory. The blue and red lines are confined and completely deconfined phases, where $N_{\mathrm{BH}} \sim O\left(N^{0}\right)$ and $N_{\mathrm{BH}}=N$, respectively. Orange lines indicate the partially deconfined phase.
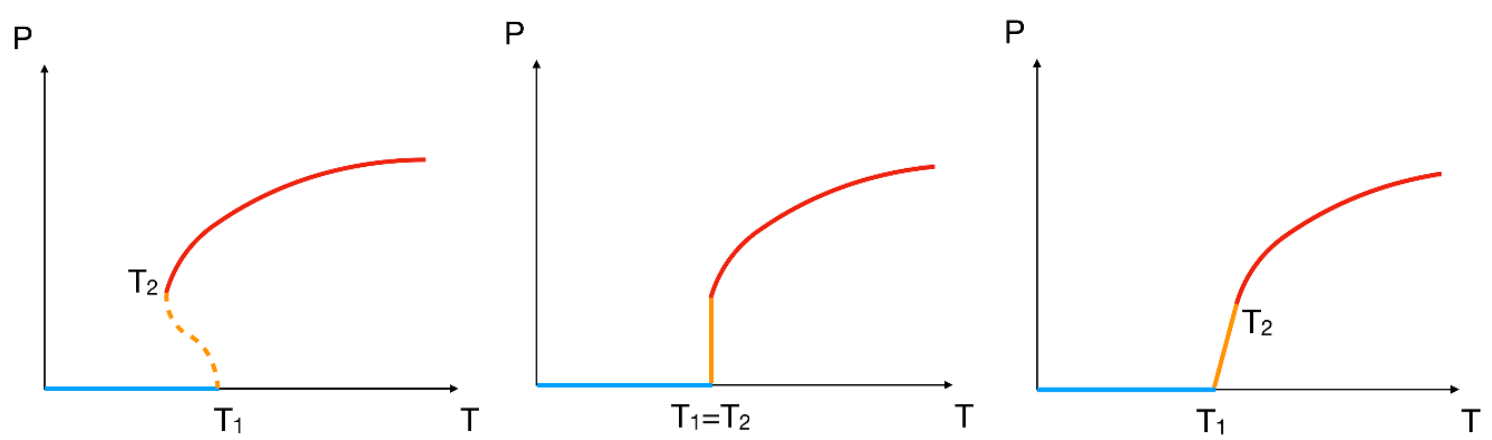

Figure 4. Cartoon pictures of the Polyakov loop $P$ as a function of temperature. The blue, orange and red lines represent the confined, partially deconfined and completely deconfined phases. Similar curves are obtained by using $E / N^{2}$ as the vertical axis as well.

- If the inflow/outflow wins when $N_{\mathrm{BH}}$ is varied slightly downwards/upwards from the saddle, the saddle is stable. In the right panels of figure 3 and figure 4 , only stable saddles exist. In $4 \mathrm{~d} \mathcal{N}=4 \mathrm{SYM}$, there is no counterpart of the right panels.

The unstable saddle exhibits the negative specific heat because at higher temperature each open string mode can be excited more (each ant contributes to more pheromone) and strong enough attraction (strong enough pheromone) resisting the emission of D-branes (outflow of the ants) can be achieved with smaller value of $N_{\mathrm{BH}}$.

Despite the similarity in the dynamics and qualitative aspects of the phase structures, there is a difference as well: $N_{\text {trail }} / N$ can be one only at $p=\infty$, namely the 'complete deconfinement' is missing in the ant theory. In appendix B.1, we show a similar model (Ant-Man model) which does capture the complete deconfinement.

Because it is not easy to see the value of $N_{\mathrm{BH}}$ manifestly in gauge theory, we need more evidence to justify the picture shown in figure 3. Later in this paper, we will provide the evidence. Meanwhile, let's focus on figure 4 first, because is rather well-established. $4 \mathrm{~d}$ $\mathcal{N}=4 \mathrm{SYM}$ on $\mathrm{S}^{3}$ has the same pattern as figure 4 , when the coupling constant $\lambda=g_{\mathrm{YM}}^{2} N$ is varied: as the coupling constant becomes smaller, the hysteresis becomes weaker $\left(T_{2}-T_{1}\right.$ becomes smaller) and phase structure becomes like the center of figure $4\left(T_{1}=T_{2}\right)[5,16]$. 
There are plenty of other examples which exhibit this pattern shown in figure 4, including:

- The real-world QCD has the rapid cross-over which resembles the right panel. By dialing the mass of the quark to sufficiently large values, the first order transition like the left panel can be realized.

- We can also consider QCD at finite baryon chemical potential. It is widely believed that the first order transition shows up at sufficiently large baryon chemical potential. $^{8}$

- The bosonic version of the plane wave matrix model, which we will study in detail in section 4.4 .

- In certain super Yang-Mills theories, the 'deconfinement' along the spatial circle is related to the black hole/black string topology change. In this case the existence of the unstable saddle (non-uniform black string) can be shown from the gravity side. We will discuss the detail in section 4.5.

Based on these examples and the arguments in this section pertaining to collective motion with positive feedback, we propose that the partial deconfinement is a generic feature of gauge theory. In section 4, we provide quantitative evidence based on the distribution of the Polyakov line phases.

\section{Quantitative tests with Polyakov line phases}

In this section we show the evidence of the partial deconfinement in various settings, both at weak coupling and strong coupling, based on the distribution of the Polyakov line phases. The relation (2.1) plays the central role. We find the universal behavior regardless of the choice of the theory.

Firstly we give a heuristic derivation of (2.1). In the picture of the partial deconfinement, $T=T_{2}$ is the lowest possible temperature in the $\mathrm{SU}(N)$ theory with the coupling constant $g_{\mathrm{YM}}^{2}$ at which all the D-branes can form a single bound state [6]. Namely, if the temperature is below $T_{2}$, some D-branes are emitted from the bound state. Next let us consider a bound state consisting of $N_{\mathrm{BH}}$ of $N$ D-branes, which describes the partially deconfined phase. We assume that the dynamics of the bound state can be described by $\mathrm{SU}\left(N_{\mathrm{BH}}\right)$ theory, neglecting $N-N_{\mathrm{BH}} \mathrm{D}$-branes outside the bound state. Then, the temperature of the bound state is identified with the lowest possible temperature in the $\mathrm{SU}\left(N_{\mathrm{BH}}\right)$ theory with the coupling constant $g_{\mathrm{YM}}^{2}$ for which $N_{\mathrm{BH}}$ D-branes can form a single bound state [6]. Hence $N_{\mathrm{BH}}$ of the $N$ Polyakov line phases are distributed as $\rho_{\text {deconfine }}(\theta)$, while the rest follow $\rho_{\text {confine }}(\theta)=\frac{1}{2 \pi}$.

An implicit assumption here is that $\rho_{\text {deconfine }}(\theta)$ does not depend on the effective 't Hooft coupling $g_{\mathrm{YM}}^{2} M$. This assumption is satisfied for the examples we consider below: in $4 \mathrm{~d}$ theories at weak coupling, the assumption can be confirmed by explicit calculations; in

\footnotetext{
${ }^{8}$ Usually the chiral transition, which can be different from deconfinement in general, is considered. See section 5 regarding this point.
} 
theories in less than four spacetime dimensions the 't Hooft coupling is dimensionful and hence it simply sets the unit of the energy scale. (In case this assumption is not correct, we need to take into account the coupling dependence of $\rho_{\text {deconfine }}(\theta)$.)

In all the cases we will study below, the transition between the partially and completely deconfined phases is the Gross-Witten-Wadia transition [17-19]. We conjecture that more broadly, the partial-to-full deconfinement transition is universally described by Gross-Witten-Wadia.

\section{$4.14 \mathrm{~d} \mathcal{N}=4 \mathrm{SYM}$ on $\mathrm{S}^{3}$}

We begin with $4 \mathrm{~d} \mathcal{N}=4 \mathrm{SYM}$ on $\mathrm{S}^{3}$. In the weak coupling limit, the phase distribution along the vertical orange line (i.e. 'Hagedorn string' [20]) has been obtained analytically. The result is $[5,16]$

$$
\rho(\theta)=\frac{1}{2 \pi}\left(1+\frac{2}{\kappa} \cos \theta\right)
$$

where $\kappa=2$ and $\kappa=\infty$ correspond to the intersection of the orange line with the red and blue lines. If the orange line were not exactly vertical, these intersections would occur at the temperatures $T=T_{2}$ and $T=T_{1}$. One obtains a phase distribution of the same form as (4.1) for the unstable saddle which appears at finite coupling, as long as the coupling is sufficiently small. ${ }^{9}$ We can rewrite $\rho(\theta)$ as

$$
\frac{1}{2 \pi}\left(1+\frac{2}{\kappa} \cos \theta\right)=\left(1-\frac{2}{\kappa}\right) \cdot \frac{1}{2 \pi}+\frac{2}{\kappa} \cdot \frac{1}{2 \pi}(1+\cos \theta)
$$

and by identifying

$$
\frac{M}{N}=\frac{2}{\kappa},
$$

we obtain (2.1), where $\rho_{\text {deconfinement }}(\theta)$ is the $\rho(\theta)$ at $\kappa=2$ :

$$
\rho_{\text {deconfinement }}(\theta)=\frac{1}{2 \pi}(1+\cos \theta) .
$$

The transition at $\kappa=2$ is the so-called Gross-Witten-Wadia transition.

Therefore it is consistent with the partial deconfinement: the Hagedorn string can be identified with the partially deconfined phase. This identification, combined with the interpretation of black hole as long string [5, 16, 21-24], naturally suggests that the unstable saddle (10d Schwarzschild black hole) at strong coupling is also in the partially deconfined phase.

As discussed in ref. [6], the partial deconfinement can explain the equation of state of the small black hole, $E \sim N^{2} T^{-7}$, and also other nontrivial features shown in the left panel of figure 1. To make the paper self contained, we explain more details in appendix A.

\footnotetext{
${ }^{9}$ In ref. [5], the partition function in the free limit is expressed by using $u_{n}=\int d \theta \rho(\theta) e^{i n \theta}$, as $Z=$ $\int d u_{n} d \bar{u}_{n} e^{-N^{2} \sum_{n \geq 1} c_{n}\left|u_{n}\right|^{2}}$. At the critical temperature, $c_{n}>0$ for $n \geq 2, c_{1}=0$, and hence $u_{n}=0$ for $n \geq 2$ and $u_{1}$ can take any value between 0 and 1 , which leads to (4.1). At weak but finite coupling, the values of $c_{n}$ change, and higher order terms such as $\left|u_{n}\right|^{4}$ can appear. Still, $c_{n}>0$ holds for $n \geq 2$, and hence $u_{n}=0$ for $n \geq 2$ regardless of the detail. Therefore, (4.1) also holds along the unstable saddle.
} 


\subsection{Other $4 \mathrm{~d}$ theories on $\mathrm{S}^{3}$}

As discussed in ref. [5], $4 \mathrm{~d}$ theories on the small three sphere can also behave like the right panel of figure 4, depending on the details of the theories. The transitions are of the second order and third order at $T=T_{1}$ and $T=T_{2}$, respectively. Again, the latter is the Gross-Witten-Wadia transition. The Polyakov line phase distribution becomes as follows:

$$
\rho(\theta)=\left\{\begin{array}{cc}
\frac{1}{2 \pi} & \left(T \leq T_{1}\right) \\
\frac{1}{2 \pi}\left(1+\frac{2}{\kappa} \cos \theta\right) & \left(T_{1}<T<T_{2}\right) \\
\frac{2}{\pi \kappa} \cos \frac{\theta}{2} \sqrt{\frac{\kappa}{2}-\sin ^{2} \frac{\theta}{2}}\left(T \geq T_{2},|\theta|<2 \arcsin \sqrt{\kappa / 2}\right)
\end{array}\right.
$$

For $T \geq T_{2}, \rho(\theta)=0$ at $2 \arcsin \sqrt{\kappa / 2} \leq|\theta| \leq \pi$. The parameter $\kappa$ is 2 at $T=T_{2}$ and $\infty$ at $T=T_{1}$. At $T \leq T_{1}$, the system is confined. If we interpret the transition at $T=T_{2}$ as separating the partially and completely deconfined phases, it is consistent with the relation (2.1), just as in section 4.1 .

\subsection{4d pure Yang-Mills on flat space}

Deconfinement transition in 4d pure Yang-Mills on flat space has been studied extensively on lattice. With a lattice regularization, the need for the renormalization makes the determination of the phase distribution tricky, namely the 'bare' phases observed in the simulation is not necessarily physical; see e.g. [25]. In refs. [26, 27], 4d pure $\mathrm{SU}(N)$ Yang-Mills theory (from $N=3$ to $N=6$ ) has been studied numerically on lattice, and the renormalization has been performed for the Polyakov loop expectation value $\langle P\rangle=\int d \theta e^{i \theta} \rho(\theta)$, so that the zero-point energy is zero. At $T=T_{2}$, the value is close to $\frac{1}{2}$, which is consistent with the GWW-ansatz described above. (Regarding this obaservation, see refs. [28, 29] for the argument closely related to ours.)

\subsection{Matrix quantum mechanics}

Matrix quantum mechanics (i.e. (0+1)-dimensional Yang-Mills) is ultraviolet finite, and is an ideal test bed for numerically studying the Polyakov loop expectation value on the lattice, without a subtlety of the renormalization.

From unstable to stable with plane wave deformation. Let us consider the bosonic analogue of the D0-brane matrix model [30-33], whose Lagrangian is given by

$$
L=N \operatorname{Tr}\left(\frac{1}{2} \sum_{I=1}^{9} D_{t} X_{I}^{2}+\frac{1}{4} \sum_{I, J=1}^{9}\left[X_{I}, X_{J}\right]^{2}\right)
$$

Here $X_{I}(I=1,2,, \cdots, 9)$ are $N \times N$ Hermitian matrices and $D_{t} X_{I}=\partial_{t} X_{I}-i\left[A_{t}, X_{I}\right]$ is the covariant derivative of $X_{I}$. This model does not exhibit a first order transition; rather, like the right panel of figure 4 , the model possesses a phase transition at $T_{1}$ (between the blue and orange lines) and another at $T_{2}$ (between the orange and red lines). The Polyakov line phases can be numerically well-fit by (4.5) [34]. 

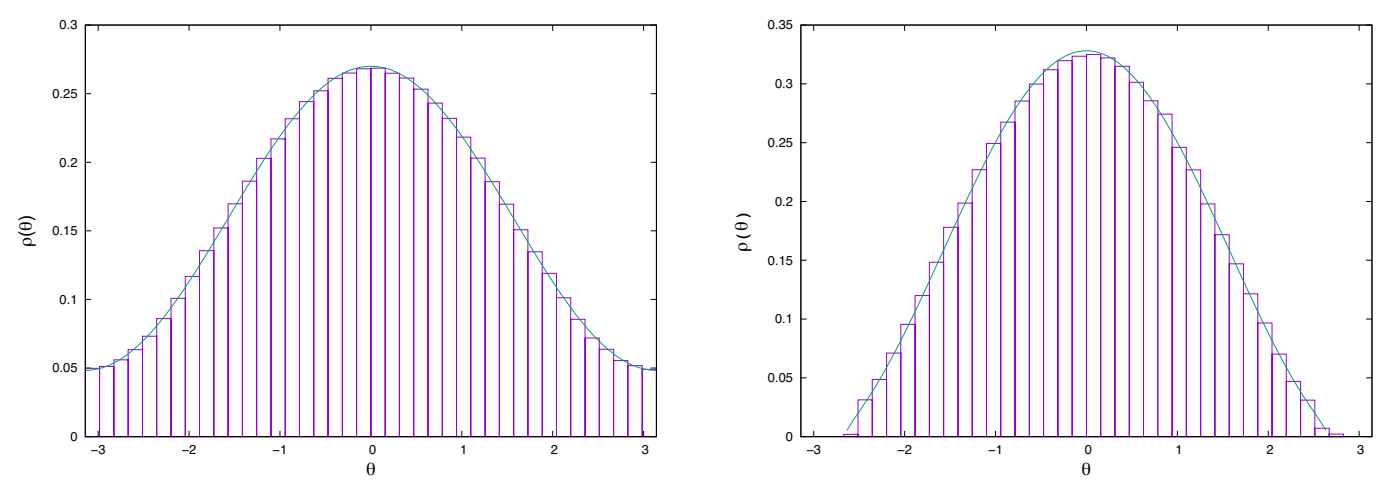

Figure 5. The distribution of Polyakov line phases. [Left] $\mu=3.0$, where the partially deconfinement phase is stable. We have plotted the distribution at $N=32$, the number of lattice points $L=18$, and temperature $T=1.15$, which is in the middle of the partially deconfined phase. The fit is $\rho(\theta)=\frac{1}{2 \pi}\left(1+\frac{2}{\kappa} \cos \theta\right)$ with $\kappa=2.87$. [Right] $\mu=5.0$, where the partially deconfinement phase is unstable and the transition is of first order. We have plotted the distribution at $N=128$, $L=16, T=1.54$, which is very close to $T_{2}$. The fit is $\rho(\theta)=\frac{2}{\pi \kappa} \cos \frac{\theta}{2} \sqrt{\frac{\kappa}{2}-\sin ^{2} \frac{\theta}{2}}$ with $\kappa=1.88$.

Next let us consider the bosonic version of the plane wave matrix model [35],

$$
\begin{aligned}
L=N \operatorname{Tr}( & \frac{1}{2} \sum_{I=1}^{9}\left(D_{t} X_{I}\right)^{2}+\frac{1}{4} \sum_{I, J=1}^{9}\left[X_{I}, X_{J}\right]^{2} \\
& \left.-\frac{\mu^{2}}{2} \sum_{i=1}^{3} X_{i}^{2}-\frac{\mu^{2}}{8} \sum_{a=4}^{9} X_{a}^{2}-i \sum_{i, j, k=1}^{3} \mu \epsilon^{i j k} X_{i} X_{j} X_{k}\right) .
\end{aligned}
$$

At $\mu=0$, this is just the model (4.6) we have discussed above. As $\mu$ is turned on, the phase transition temperatures $T_{1}$ and $T_{2}$ gradually approach to each other, and eventually coincide, resembling the middle panel of figure 4. If $\mu$ is tuned even larger, the hysteresis sets in. There, the partial deconfinement phase turns to the unstable saddle. Intuitively, the reason that the first order transition emerges at large $\mu$ is that the eigenvalues of matrices (the location of D-branes) are pushed toward the origin, the off-diagonal matrix elements (open strings) can be excited more easily, and the interaction between the Dbranes becomes stronger.

At small $\mu$ region, we can numerically show that the GWW ansatz (4.5) works, just in the same way as $\mu=0$; see the left panel of figure 5 . At $\mu \sim 5$ the first order transition sets in. By using the lattice regularization explained in appendix C, with SU(128) gauge group and 16 lattice sites, the hysteresis can be seen around $T=1.55$. As expected, in the deconfinement phase $\rho(\theta)$ is consistent with the last row of (4.5), and toward the endpoint $\left(T=T_{2}\right)$ the value of $\kappa$ approaches 2 and the gap closes; see the right panel of figure 5 .

D0-brane quantum mechanics. Now we consider the full, supersymmetric D0-brane matrix model [30-33]. ${ }^{10}$ With the plane wave deformation $(\mu>0)$ [35], this theory is

\footnotetext{
${ }^{10}$ This part is based on forthcoming work with the Monte Carlo String/M-theory Collaboration. The detail will appear in near future, as a part of a separate publication.
} 
expected to have a first order confinement/deconfinement transition (see e.g. ref. [36]). At $\mu=0$, there are two possibilities: (i) the first order transition survives all the way down to $\mu=0$, or (ii) it disappears at finite $\mu$.

In the case of (i), the transition temperature should be zero at $\mu=0$, and the confinement phase should disappear, as in the flat space limit of $4 \mathrm{~d} \mathcal{N}=4 \mathrm{SYM}$. Then it is natural to expect the Hagedorn growth exactly at $T=0$, as depicted in the center panel of figure 4 , with $T_{1}=T_{2}=0$. On the other hand, in ref. [37] it has been argued that the Polyakov line phases should be almost uniformly distributed and behave like (4.1). Then the option (ii), namely the right panel of figure 4 with $T_{1}=0$ and $T_{2}>0$, is the natural candidate. ${ }^{11}$

To explore the above possibilities, we can use the simulation data obtained from the previous [38, 39] and ongoing projects by the Monte Carlo String/M-theory Collaboration. Unfortunately, this data only covers a moderately high temperature region for which $\langle|P|\rangle \gtrsim 0.7$, and hence we cannot judge which possibility is correct. However, we did confirm that the GWW-ansatz holds for the high temperature phase $\left(T \geq T_{2}\right)$.

\subsection{2d maximal SYM and black hole/black string topology change}

With the Euclidean signature, the deconfinement transition is the breakdown of the center symmetry along the temporal circle. Mathematically, it is the same as the center symmetry along the spatial circle, when (one or more of) the spatial dimensions are compactified. ${ }^{12}$ The 'spatial deconfinement' - the breakdown of the center symmetry along the spatial circle - can also be explained by the framework of the partial deconfinement.

Consider 2d maximal SYM on spatial circle. It is dual to a system of D1-branes in type IIB string theory [33], which is equivalent to a system of D0-branes in type IIA string theory via T-duality. In the D0-brane picture, there can exist a string-like distribution of D0-branes - called a "black string" — wrapping around the spatial circle. There can also be black holes, which correspond to a localized distribution of D0 branes. The distribution of D0-branes is described by the phase distribution of the spatial Polyakov loop. Thus, we can relate the phase structure of SYM and the black hole/black string system [40].

There can be 'confined', 'partially deconfined' and 'completely deconfined' phases, which correspond to the uniform black string, non-uniform black string and black hole, respectively. ${ }^{13}$ When the non-uniform black string turns into a black hole, the Polyakov line phase distribution $\rho(\theta)$ develops a gap. This is consistent with the partial deconfinement picture.

At low temperatures, the dual gravity description is weakly coupled, and a detailed analysis can be done with numerical relativity. There are two stable phases — black hole

\footnotetext{
${ }^{11}$ Ref. [36] also discussed the possibility that the first order transition disappears at small but finite $\mu$.

${ }^{12}$ The temporal circle has a periodic boundary condition for bosons and an anti-periodic boundary condition for fermions. Below, for the spatial circle, we impose periodic boundary conditions for both bosons and fermions.

${ }^{13}$ Visually the ant trail would look like black string, but actual correspondence is a little bit counterintuitive. Thick, stable ant trail is black hole while the thin trail is non-uniform black string. The disappearance of the ant trail is the formation of the uniform black string.
} 
and uniform black string - separated by the first order phase transition, and they are connected by the unstable saddle — nonuniform black string - [40-44]. The instability toward the deconfined phase is the gauge theory analogue of the Gregory-Laflamme instability [45]. Also, as mentioned above, the gap at $\theta= \pm \pi$ appears exactly at the border between the black hole and non-uniform black string phases. These can naturally be explained by the partial deconfinement.

At high temperatures, we can use the one-dimensional bosonic matrix model (4.6) to describe the thermal properties of the system. In this regime, all three phases appear as stable saddles, and the phase distribution [34] is consistent with the partial deconfinement, as we have already seen in section 4.4.

\section{Conclusion and discussions}

In this paper, we have argued that the confined and deconfined phases in gauge theories are connected by a partially deconfined phase, which can be stable or unstable depending on the details of the theory. When this phase is unstable, it is the gauge theory counterpart of the small black hole phase in the dual string theory. Furthermore, we have argued that partial deconfinement is closely related to the Gross-Witten-Wadia (GWW) transition. In order to understand the mechanism behind the partial deconfinement intuitively, we have advocated the similarity between the dynamics of gauge theories and the collective behaviors of ants. The quantitative evidence is obtained by comparing the distribution of Polyakov line phases with (2.1).

A natural, immediate question is whether the partial deconfinement can be seen in the experiments, especially in QCD. There are a few subtleties in the application of the notion of the partial deconfinement to real-world QCD. Firstly, $N=3$ may not be so large. Also the existence of the matter in the fundamental representation, which explicitly breaks the center symmetry, may modify the nature of the transition. Let us still consider possible implications, keeping these subtleties in mind.

- The QCD phase transition at zero chemical potential appears to be a rapid crossover [46], which resembles the right panel of figure 4. By changing the quark mass, the first order transition (the left panel of figure 4) can also be realized. Hence it is natural to expect that the crossover region contains counterparts of partially and completely deconfined phases. The partial deconfinement would lead to a new kind of spectrum which has not been considered so far, and hence would be phenomenologically relevant.

- It is widely believed that, at finite chemical potential, the transition becomes first order. (See e.g. [47] for a review.) If this is true, it is natural to expect that the partially deconfined unstable phase - the gauge theory counterpart of the Schwarzschild black hole - exists. It would be a useful setting for 'experimental realization' of evaporating black hole! Note that the stringy correction should be large and hence it would not be very close to the black hole in weakly coupled gravity. Nonetheless, it would be interesting if some universal features could be seen. 
- A few remarks regarding the nature of the phase transition at finite chemical potential is in order here. ${ }^{14}$

Usually it is argued that the chiral transition (characterized by the growth of chiral condensate from $\simeq 0$ to a large value), which can be different from deconfinement (characterized by the growth of the Polyakov loop) in general, becomes first order. Whether these two transitions are correlated as $\mu=0$ case is not clear because the lattice QCD simulation is difficult due to the sign problem.

Whether two transitions coincide or not, the unstable phase can exist as long as there is a hysteresis. At a possible first order chiral transition, if the Polyakov loop jumps as well - whether zero to nonzero, or nonzero to nonzero - the argument for the small black hole phase presented in section 3 would apply without change. ${ }^{15}$ Note that, in the case of $4 \mathrm{~d} \mathcal{N}=4$ super Yang-Mills, the small black hole phase connected the completely deconfined and Hagedorn string phases, both correspond to $P>0$.

- Given that we might be able to see the 'evaporating black hole' experimentally, it is interesting to study possible experimental signals associated with the first order phase transition. The nature of the phase transitions discussed so far looks rather universal. Hence it would be useful to consider 'applied AdS/CFT' based on 4d SYM on $\mathrm{S}^{3}$ (see e.g. ref. [48]).

There are several other issues which are important from the quantum gravity point of view:

- Analyses of the gravitational dual of 4d maximal SYM suggests the existence of a first order transition corresponding to the localization of the black hole along $\mathrm{S}^{5}$, even in the microcanonical treatment $[49,50]$. This cannot be explained by the gauge theory argument in this paper. We emphasize that the color degrees of freedom would play a crucial roles in this case as well. One possibility is that a GWW-like transition in the microcanonical treatment describes the transition between the positive and negative specific heat parameter regions of the $\mathrm{AdS}_{5}$ black hole which are not localized along $\mathrm{S}^{5}$, and the transition associated with the localization along $\mathrm{S}^{5}$ exists separately. It may well be the case that the localization along $\mathrm{S}^{5}$ (i.e. breaking of the R-symmetry) corresponds to the GWW transition. That the previous weak-coupling analyses did not capture this transition would mean that the stringy corrections resolve it.

- When refs. $[9,10]$ discussed the negative specific heat in the context of gauge/gravity duality, the D0-brane matrix model has been considered as a concrete example. Instead of the confinement, the Higgs mechanism associated with the emission of the eigenvalues due to the flat direction in the moduli has been used for the reduction of the unlocked degrees of freedom. For the flat direction to exist, supersymmetry is crucial, and hence the generalization of the argument to nonsupersymmetric systems,

\footnotetext{
${ }^{14}$ We would like to thank K. Fukushima for useful comments regarding these points.

${ }^{15} \mathrm{~A}$ subtlety here is that the Polyakov loop may not be a good quantity to characterize the partial deconfinement, because of the existence of the fundamental fermion.
} 
like our universe, was not clear. The partial deconfinement is almost the same (i.e. some degrees of freedom are confined rather than Higgsed) but supersymmetry is not required.

- The numerical tests explained in section 4 did not directly consider the unstable saddle. In principle, we could directly see the unstable saddle of the path integral (1.1), by counting the number of Monte Carlo samples for each value of $E$ and determining $F(E, T)$ in (1.1). Of course, a naïve simulation samples only the configurations around the minima, but we might be able to circumvent this problem by restricting the energy to a narrow band. ${ }^{16,17}$ Whether such a sampling procedure works with reasonable computational resources is not clear at this moment, but it is certainly worth trying. It is also important to derive the negative specific heat analytically, away from the weak coupling region. See ref. [54] for a recent attempt.

Last but not least, the correspondence between the ant trail and black hole is not perfect. The biggest difference is that $N_{\text {trail }}$ can reach $N$ only at $p=\infty$, and as a consequence, a GWW-like transition is missing. It would be interesting if there is a more precise analogue of black holes which exhibits the GWW transition. See an improved model discussed in appendix B.1 (the Ant-Man model) for an attempt along this direction.

\section{Acknowledgments}

We thank Tatsuo Azeyanagi, David Berenstein, Ramy Brustein, Jordan Cotler, Oscar Dias, Pau Figueras, Kenji Fukushima, Hrant Gharibyan, Yoshimasa Hidaka, Juan Maldacena, Marco Panero, Rob Pisarski, Enrico Rinaldi, Jorge Santos, Bo Sundborg, Masaki Tezuka and Naoki Yamamoto for discussions and comments. We thank Jordan Cotler also for carefully reading the manuscript many times and providing us with countlessly many comments. M. H. thanks Brown University for the hospitality during his stay while completing the paper, and acknowledges the STFC Ernest Rutherford Grant ST/R003599/1. M. H. and G. I. acknowledge JSPS KAKENHI Grants 17K14285 and 16K17679, respectively. The work of G. I. was also supported, in part, by Program to Disseminate Tenure Tracking System, MEXT, Japan.

We originally learned about the ant model from ref. [55]. We thank British people's devotion to the beautiful game of football, which made this kind of book commercially successful.

\footnotetext{
${ }^{16}$ Essentially the same manipulation for the path integral in the gravity side has been considered in ref. [51].

${ }^{17}$ Such procedure is straightforward for the theories without the sign problem. In many supersymmetric theories, the sign problem does exist, namely the determinant of the Dirac operator is complex, and hence we need to find a way to fix the energy taking into account the effects of the complex phase. Fortunately, the complex phase is often close to 1 , so that it can simply be neglected (see ref. [52] for recent review). Even when the complex phase fluctuates violently, it is not correlated to various physical quantities and can be neglected, at least in the case of D0-brane matrix model $[38,53]$. Therefore, there is a fair chance that such strategy can work even for supersymmetric theories which have good weakly-coupled gravity duals simply neglecting the phase.
} 


\section{A Small black hole from gauge theory}

In this appendix, we review the heuristic explanation of the black hole equation of state from the dual $4 \mathrm{~d}$ super Yang-Mills. We closely follow ref. [6]. The most basic assumption of the partial deconfinement introduced in ref. [6] is that, regardless of the value of $N_{\mathrm{BH}}$, the property of the small black hole (unstable saddle) is the same as the one at $T=T_{2}$ and $N_{\mathrm{BH}}=N$. Let $X_{\mathrm{BH}}$ be the $N_{\mathrm{BH}} \times N_{\mathrm{BH}}$ sub-matrix of the scalar $X$, which corresponds to the partially deconfined $\mathrm{SU}\left(N_{\mathrm{BH}}\right)$. As $N_{\mathrm{BH}}$ changes, the 't Hooft coupling effectively changes as $g_{\mathrm{YM}}^{2} N_{\mathrm{BH}}=\left(g_{\mathrm{YM}}^{2} N\right) \cdot\left(N_{\mathrm{BH}} / N\right)=\lambda \cdot\left(N_{\mathrm{BH}} / N\right) \equiv \lambda_{\mathrm{BH}}$.

When $\lambda_{\mathrm{BH}} \ll 1$, the $N_{\mathrm{BH}} \times N_{\mathrm{BH}}$ block is weakly coupled, and hence we simply see the Hagedorn growth. On the other hand, when $\lambda_{\mathrm{BH}} \gg 1$, the $N_{\mathrm{BH}} \times N_{\mathrm{BH}}$ block is strongly coupled. Then the potential term $\frac{1}{g_{\mathrm{YM}}^{2}} \operatorname{Tr}\left[X_{\mathrm{BH} I}, X_{\mathrm{BH} J}\right]^{2}=\frac{N_{\mathrm{BH}}}{\lambda_{\mathrm{BH}}} \operatorname{Tr}\left[X_{\mathrm{BH} I}, X_{\mathrm{BH} J}\right]^{2}$ is more important than the kinetic term of the action. By using $Y_{\mathrm{BH}} \equiv X_{\mathrm{BH}} / \lambda_{\mathrm{BH}}^{1 / 4}$, the potential becomes independent of the coupling constant. As mentioned in section 4, we identify the temperature $T$ to be the lowest possible temperature in the $\mathrm{SU}\left(N_{\mathrm{BH}}\right)$ theory at which all $N_{\mathrm{BH}}$ D-branes can form the bound state. Given that there is no particular $\lambda_{\mathrm{BH}}$ dependence in terms of $Y_{\mathrm{BH}}$, it is natural to suppose that the eigenvalues of $Y_{\mathrm{BH}}$ at $T$ are independent of $\lambda_{\mathrm{BH}}$, and hence the eigenvalues of $X_{\mathrm{BH}}$ scale as $\lambda_{\mathrm{BH}}^{1 / 4}$. Intuitively, the eigenvalue of $X_{\mathrm{BH}}$ corresponds to the radius of the black hole, which sets a natural inverse energy scale. So it is natural to expect $T \sim \lambda_{\mathrm{BH}}^{-1 / 4} \sim\left(N_{\mathrm{BH}} / N\right)^{-1 / 4}$. ${ }^{18}$ Also, from the dimensional analysis and 't Hooft counting, the energy and entropy should scale as $E \sim N_{\mathrm{BH}}^{2} T \sim N^{2}\left(N_{\mathrm{BH}} / N\right)^{7 / 4} \sim N^{2} T^{-7}$ and $S \sim N_{\mathrm{BH}}^{2} \sim N^{2}\left(N_{\mathrm{BH}} / N\right)^{2} \sim N^{2} T^{-8}$. (The 't Hooft counting alone cannot fix the dependence on the 't Hooft coupling. Here we implicitly assumed that it is independent of the coupling in the strongly coupled region. This is equivalent to assuming that the Newton constant is independent of $\lambda$ in the dual frame $\left(R_{\mathrm{S}^{3}}\right.$ and $R_{\mathrm{AdS}}$ fixed), which can be confirmed analytically in the supersymmetrypreserving setups.)

Next let us consider the M-theory parameter region of the ABJM theory [56]. ${ }^{19} \mathrm{We}$ take the Chern-Simons level $k$ to be $O(1)$, and send $N$ to infinity. The potential term of the action is schematically $\sim \frac{N}{\lambda} \operatorname{Tr} X^{6}\left(\lambda=\frac{N}{k}\right)$, where $X$ stands for the scalar fields in the bifundamental representation. The $\lambda$ dependence disappears if we use $Y \equiv \lambda^{-1 / 6} X$, and hence the natural scaling of the eigenvalues is $\lambda_{\mathrm{BH}}^{1 / 6}$. This motivates $T \sim\left(N_{\mathrm{BH}} / N\right)^{-1 / 6}$. Note that this is the scaling of the Planck scale in the dual frame. The Newton constant is $G_{\mathrm{N}, 11} \sim \sqrt{\lambda} / N^{2} \sim N^{-3 / 2}$, which can be checked by supersymmetric localization [57]. Hence the entropy is $S \sim N_{\mathrm{BH}}^{3 / 2} \sim N^{3 / 2} T^{-9} \sim \frac{1}{G_{\mathrm{N}, 11} T^{9}}$, and the energy is $E \sim \frac{1}{G_{\mathrm{N}, 11} T^{8}}$. This is the correct behavior in the eleven dimensional supergravity.

\footnotetext{
${ }^{18}$ Intuitively, at higher temperatures more open strings are excited, D-branes are more tightly bound, and the radius of the black hole becomes smaller. This is the same as the scaling of $\ell_{s}$ in the dual frame $\left(R_{\mathrm{S}^{3}}\right.$ and $R_{\mathrm{AdS}}$ fixed).

${ }^{19}$ Whether the same argument holds in the type IIA string theory limit (typically $N / k$ fixed) is a subtle issue, because the moduli $\left(\mathbb{C}^{4} / \mathbb{Z}_{k}\right)^{N} /$ (permutation), and the dual gravity geometry $\mathrm{AdS}_{4} \times \mathrm{S}^{7} / \mathbb{Z}_{k}$, explicitly depend on $k \sim N$. If we apply the same power counting naïvely, we obtain $E \sim \frac{1}{G_{\mathrm{N}, 10^{-8}}}$, which is not the equation of state of the ten dimensional black hole.
} 


\section{B The large $N$ limit of the ant equation}

In ref. [14], the number of the ants in the colony $N$ is varied while other parameters $\alpha, s$ and $p$ are fixed. In order to see the resemblance between the ant model and the holographic description of a black hole, it is convenient to fix $N$ to be a large value. Like in string theory and quantum field theory, we expect mathematical simplifications to arise in the natural large- $N$ (many-ant) limit. Furthermore, in the large- $N$ limit, the phase transition appears in the mathematically precise sense.

Let us divide the left and right hand sides of (3.3) by $N$. By using $x \equiv N_{\text {trail }} / N$, $\tilde{\alpha} \equiv \alpha / N$ and $\tilde{s} \equiv s / N$, we obtain

$$
\frac{d x}{d t}=(\tilde{\alpha}+p x)(1-x)-\frac{\tilde{s} x}{\tilde{s}+x} .
$$

We treat this $x$ as an order $N^{0}$ quantity. The 'ant equation' $\frac{d x}{d t}=0$ becomes

$$
f(x) \equiv p x^{3}+(\tilde{\alpha}-p+p \tilde{s}) x^{2}+(\tilde{\alpha} \tilde{s}-p \tilde{s}-\tilde{\alpha}+\tilde{s}) x-\tilde{\alpha} \tilde{s}=0 .
$$

By definition,

$$
0 \leq x \leq 1
$$

is required. Also, by 'physical' reasoning, we should impose

$$
p, \tilde{\alpha}, \tilde{s} \geq 0 .
$$

Here $p$ is the strength of the pheromone secreted by each ant. It is natural to regard $p$ to be of order $N^{0}$, as per ref. [14]. The value of $p$ can change depending on the environment around the nest. For example, if the air is dry, the pheromone can evaporate quickly and hence $p$ is smaller.

The parameter $s$ controls the rate that ants leave the trail; maybe they get bored, maybe they get tired. It is natural to assume that the number of such ants is proportional to $N$ and hence $\tilde{s}$ is of order one. This scaling of $s$ differs from the treatment in ref. [14].

Finally, the parameter $\alpha$ is the probability that each ant spontaneously find the food source without help from the pheromones. Then $\alpha$ should be almost independent of $N$, and hence, it is natural to take $\tilde{\alpha} \sim 1 / N$ as per ref. [14].

For fixed $\tilde{\alpha}$ and $\tilde{s}$, we solve (B.2) for various values of $p$ and plot $x$ as a function of $p$. Two limiting situations, $p \rightarrow 0$ and $p \rightarrow \infty$, are easy:

- When $p \rightarrow 0, \tilde{\alpha} x^{2}+(\tilde{\alpha} \tilde{s}-\tilde{\alpha}+\tilde{s}) x-\tilde{\alpha} \tilde{s}=0$, and hence $x \simeq \tilde{\alpha}$, namely $x$ is almost zero. In words: if there is no pheromone, there is no trail.

At finite $p$, the solution close to zero can be written as $x=\frac{\tilde{\alpha}}{1-p}+O\left(\tilde{\alpha}^{2}\right)$. The deviation from zero becomes large when $1-p$ becomes of order of $\tilde{\alpha}$.

- When $p \rightarrow \infty, p x^{3}+(-p+p \tilde{s}) x^{2}-p \tilde{s} x$ has to be of order one, and hence $x^{3}+$ $(-1+\tilde{s}) x^{2}-\tilde{s} x$ has to be almost zero. Then $x \rightarrow 1$. ( $x \rightarrow 0$ may appear to be fine as well, but it requires $x \simeq-\frac{\tilde{\alpha}}{p}<0$ and hence is 'unphysical'. Yet another solution 
$x \simeq-\tilde{s}$ is also 'unphysical'.) Namely, if the pheromone is extremely strong, all ants join the trail.

Let us write $x=1-\epsilon$ there. Then, $\epsilon \simeq \frac{\tilde{s}}{p(\tilde{s}+1)}$.

The deviation from 'confinement' $x \sim \tilde{\alpha}$ sets in at $p \simeq 1$. If there is another solution to the ant equation there, we should see an $S$-shape curve (i.e. the first order transition). At $p=1$, the ant equation is $x^{3}-(1-\tilde{s}) x^{2} \simeq 0$. Hence, if $\tilde{s}<1$, there are two solutions near $x=0$ (analogous to 'confined' and 'partially deconfined' phases) and the other near $x=1-\tilde{s}$ (analogous to a 'completely deconfined' phase).

Let us study the shape of the $S$-curve more precisely. It is described by

$$
p x^{3}-p(1-\tilde{s}) x^{2}+(1-p) \tilde{s} x \simeq 0 .
$$

The 'partially' and 'completely' deconfined phases are

$$
x \simeq \frac{1-\tilde{s} \pm \sqrt{(1-\tilde{s})^{2}-\frac{4(1-p) \tilde{s}}{p}}}{2} .
$$

The bending point is where $\sqrt{(1-\tilde{s})^{2}-\frac{4(1-p) \tilde{s}}{p}}$ becomes zero, namely

$$
p=\frac{4 \tilde{s}}{(1-\tilde{s})^{2}+4 \tilde{s}} .
$$

As $\tilde{s}$ approaches zero, the bending point gets closer to 0 and the hysteresis becomes stronger. This behavior is 'physically' almost trivial: if no ant leaves the trail, no pheromone is needed to keep the existing trail. The small $\tilde{s}$ region is similar to the strong coupling region of $4 \mathrm{~d}$ SYM.

As $\tilde{s}$ approaches 1 , the hysteresis becomes weaker, and the unstable saddle becomes closer to the vertical straight line near $x=0$. This resembles the Hagedorn string.

For $\tilde{s}>1$, the 'partially deconfined' phase becomes stable. This is analogous to the cross-over in real-world QCD. Right above $p=1$, one of (B.6) describes this phase. By writing $p=1+q$, we obtain

$$
x \simeq \frac{q \tilde{s}}{\tilde{s}-1} .
$$

As $q \rightarrow 0$, we have that $x$ is continuous, but $\frac{d x}{d q}$ is not. Thus the transition from 'confinement' to 'partial deconfinement' is of second order in this case, just like the case of large- $N$ Yang-Mills. Note that the large- $N$ limit, which is analogous to the thermodynamic limit in statistical physics, is necessary for the phase transition to take place; see figure 6 .

Although the transition from 'confinement' to 'partial deconfinement' is nicely captured by the ant model, the transition to the 'complete deconfinement' is not very precisely captured. At $\tilde{s} \rightarrow 0$, the value of $x$ at the bending point is $1 / 2$, which is different from the value we expect in SYM, 1. At $\tilde{s}=1$, the analogue of the Hagedorn growth does not continue up to $x=1$. As we have already seen, the large- $p$ asymptotic behavior is $x \simeq 1-\frac{\tilde{s}}{p(\tilde{s}+1)}$. Hence the 'complete deconfinement' is achieved only at $p=\infty$. The same applies to $\tilde{s}>1$ as well. Hence the GWW transition, which separates the partially and 

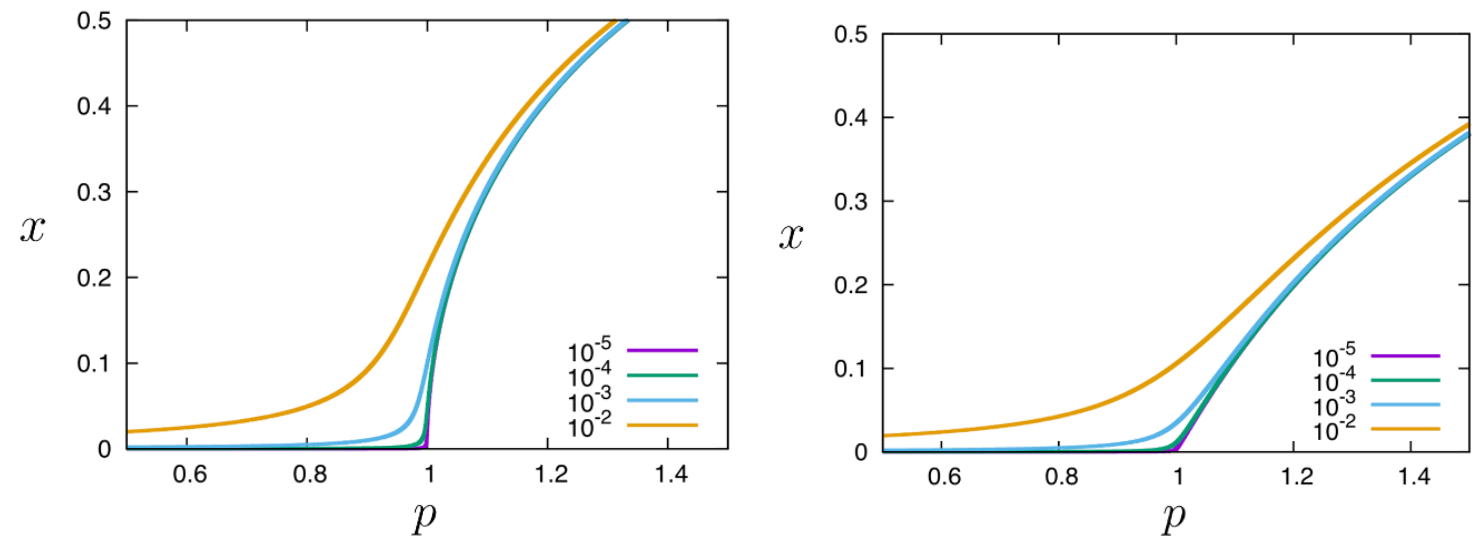

Figure 6. $x$ vs $p$ with $\tilde{s}=1$ (left) and $\tilde{s}=5$ (right), $\tilde{\alpha}=10^{-2}, 10^{-3}, 10^{-4}$ and $10^{-5}$. We can see the emergence of the singularity at $p=1$ as $\tilde{\alpha}$ approaches zero, or equivalently, in the large- $N$ limit.

completely deconfined phases, is not captured by the ant model. It would be intereting if we can find other complex systems which mimic black holes more precisely. Below, we give a toy model which captures the complete deconfinement.

\section{B.1 Modified ant model (Ant-Man model)}

Here we consider a modified ant model — 'Ant-Man model' — which gives the ants some human characteristics. We assume they think like us humans: when everybody is on the trail, they are scared to leave. If such human-ish ants existed, the ant trail model would be modified as

$$
\frac{d x}{d t}=(\tilde{\alpha}+p x)(1-x)-\frac{\tilde{s} x}{\tilde{s}+x} \cdot\left(1-x^{2}\right) .
$$

Here the factor $1-x^{2}$ multiplies to the outflow factor, so that no ant leaves the trail when all ants are on the trail $(x=1)$. Taking into account the constraint $0 \leq x \leq 1$, the phase structures become those in figure 7. They are closer to Yang-Mills (figure 3), except that new unstable trail ( $x=1$, shown by the dotted line) appears. This unwanted unstable trail is actually harmless; it can be eliminated by a small change such as $\left(1-x^{2}\right) \rightarrow\left(1+\epsilon-x^{2}\right)$ with infinitesimally small positive $\epsilon$ (i.e. a little bit of courage to leave the trail).

We emphasize that we have written down this model just to mimic Yang-Mills more closely. There is no theoretical or experimental justification of the existence of Ant-Men. Still, such models would be useful for obtaining the intuition into the nature of Yang-Mills and string theory.

\section{Monte Carlo simulation of the matrix model}

The simulation we have used for the study of the bosonic matrix model is based on the simulation code for the BMN matrix model written by M. H. for the Monte Carlo String/Mtheory collaboration. We have just removed fermions from the code. ${ }^{20}$

\footnotetext{
${ }^{20}$ The code is available upon request to $\mathrm{M} . \mathrm{H}$.
} 

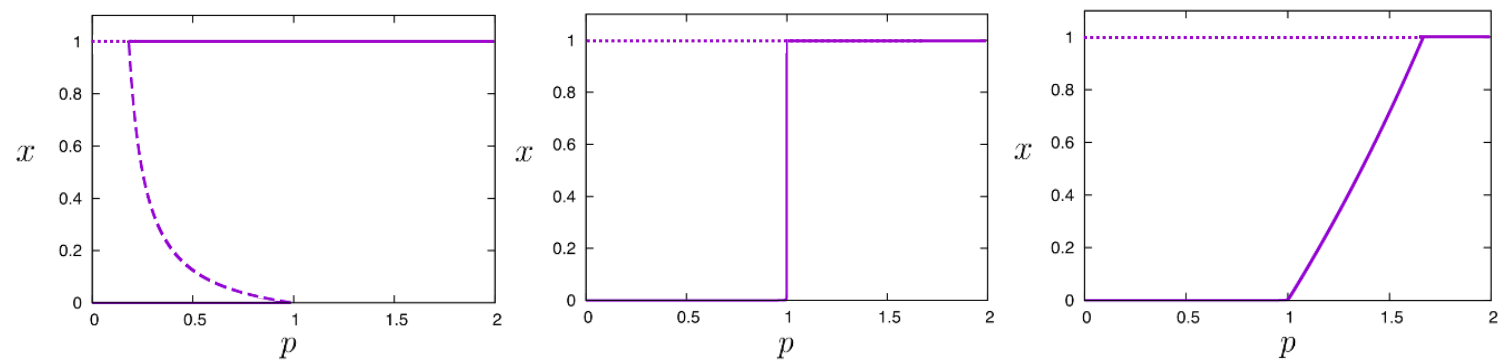

Figure 7. $x \equiv \frac{N_{\text {trail }}}{N}$ versus $p$ in the modified ant model (B.9), with $\alpha=1, \tilde{s} \equiv \frac{s}{N}=0.1$ (left), 1.0 (middle) and 5.0 (right), $N=10^{5}$.

The lattice action is

$$
\begin{aligned}
S_{\text {Lattice }}= & \frac{N}{2 a} \sum_{t, M} \operatorname{Tr}\left(D X_{M}(t)\right)^{2}-\frac{N a}{4} \sum_{t, M, N} \operatorname{Tr}\left[X_{M}(t), X_{N}(t)\right]^{2} \\
& +a N \sum_{t} \operatorname{Tr}\left\{\frac{\mu^{2}}{2} \sum_{i=1}^{3} X_{i}(t)^{2}+\frac{\mu^{2}}{8} \sum_{a=4}^{9} X_{a}(t)^{2}+i \sum_{i, j, k=1}^{3} \mu \epsilon^{i j k} X_{i}(t) X_{j}(t) X_{k}(t)\right\} \\
& -\sum_{i<j} 2 \log \left|\sin \left(\frac{\alpha_{i}-\alpha_{j}}{2}\right)\right|
\end{aligned}
$$

where $U=\operatorname{diag}\left(e^{i \alpha_{1} / N_{t}}, e^{i \alpha_{2} / N_{t}} \cdots, e^{i \alpha_{N} / N_{t}}\right),-\pi \leq \alpha_{i}<\pi$, and

$$
D X(t) \equiv \frac{1}{2} U^{2} X(t \pm 2 a)\left(U^{\dagger}\right)^{2}+2 U X(t \pm a) U^{\dagger}-\frac{3}{2} X(t)=a D_{t} X(t)+O\left(a^{3}\right) .
$$

We simulate this action by using the Hybrid Monte Carlo algorithm.

The Polyakov loop is defined by

$$
P=\frac{1}{N} \sum_{j=1}^{N} e^{i \alpha_{j}} .
$$

The phases are $\alpha_{1}, \alpha_{2}, \cdots, \alpha_{N}$. We need to take into account the ambiguity of the global $\mathrm{U}(1)$ factor, with which all $\alpha$ 's are shifted by a constant. We fix it by requiring $P=|P|$.

We have studied $\mu=1,2,3$ and 4 with matrix size $N=32$, lattice size $L=18$. In this region, we obtained the data consistent with the existence of stable partially deconfined phase, although a first order transition may emerge at larger $N$. We have also studied $\mu=5$ with $N=128$ and $L=16$. There, we have observed a clear hysteresis around $T=1.55$; namely, two different phases are observed with hot start (lower the temperature gradually) and cold start (raise the temperature gradually).

Open Access. This article is distributed under the terms of the Creative Commons Attribution License (CC-BY 4.0), which permits any use, distribution and reproduction in any medium, provided the original author(s) and source are credited. 


\section{References}

[1] G. 't Hooft, Dimensional reduction in quantum gravity, Conf. Proc. C 930308 (1993) 284 [gr-qc/9310026] [INSPIRE].

[2] L. Susskind, The world as a hologram, J. Math. Phys. 36 (1995) 6377 [hep-th/9409089] [INSPIRE].

[3] J.M. Maldacena, The large $N$ limit of superconformal field theories and supergravity, Int. J. Theor. Phys. 38 (1999) 1113 [hep-th/9711200] [INSPIRE].

[4] E. Witten, Anti-de Sitter space, thermal phase transition and confinement in gauge theories, Adv. Theor. Math. Phys. 2 (1998) 505 [hep-th/9803131] [InSPIRE].

[5] O. Aharony, J. Marsano, S. Minwalla, K. Papadodimas and M. Van Raamsdonk, The Hagedorn - deconfinement phase transition in weakly coupled large $N$ gauge theories, Adv. Theor. Math. Phys. 8 (2004) 603 [hep-th/0310285] [INSPIRE].

[6] M. Hanada and J. Maltz, A proposal of the gauge theory description of the small Schwarzschild black hole in $A d S_{5} \times S^{5}$, JHEP 02 (2017) 012 [arXiv: 1608.03276] [INSPIRE].

[7] D. Berenstein, Submatrix deconfinement and small black holes in AdS, JHEP 09 (2018) 054 [arXiv: 1806.05729] [INSPIRE].

[8] C.T. Asplund and D. Berenstein, Small AdS black holes from SYM, Phys. Lett. B 673 (2009) 264 [arXiv:0809.0712] [INSPIRE].

[9] E. Berkowitz, M. Hanada and J. Maltz, Chaos in Matrix Models and Black Hole Evaporation, Phys. Rev. D 94 (2016) 126009 [arXiv:1602.01473] [INSPIRE].

[10] E. Berkowitz, M. Hanada and J. Maltz, A microscopic description of black hole evaporation via holography, Int. J. Mod. Phys. D 25 (2016) 1644002 [arXiv:1603.03055] [INSPIRE].

[11] J. Liddle and M. Teper, The deconfining phase transition in $D=2+1 \mathrm{SU}(N)$ gauge theories, arXiv:0803.2128 [INSPIRE].

[12] B. Lucini, M. Teper and U. Wenger, The high temperature phase transition in $\mathrm{SU}(N)$ gauge theories, JHEP 01 (2004) 061 [hep-lat/0307017] [INSPIRE].

[13] L. Álvarez-Gaumé, P. Basu, M. Mariño and S.R. Wadia, Blackhole/String Transition for the Small Schwarzschild Blackhole of $A d S_{5} x S^{5}$ and Critical Unitary Matrix Models, Eur. Phys. J. C 48 (2006) 647 [hep-th/0605041] [INSPIRE].

[14] M. Beekman, D.J.T. Sumpter and F.L.W. Ratnieks, Phase transition between disordered and ordered foraging in Pharaoh's ants, Proc. Nat. Acad. Sci. 98 (2001) 9703.

[15] L. Kofman, A.D. Linde, X. Liu, A. Maloney, L. McAllister and E. Silverstein, Beauty is attractive: Moduli trapping at enhanced symmetry points, JHEP 05 (2004) 030 [hep-th/0403001] [INSPIRE].

[16] B. Sundborg, The Hagedorn transition, deconfinement and $N=4$ SYM theory, Nucl. Phys. B 573 (2000) 349 [hep-th/9908001] [INSPIRE].

[17] D.J. Gross and E. Witten, Possible Third Order Phase Transition in the Large N Lattice Gauge Theory, Phys. Rev. D 21 (1980) 446 [inSPIRE].

[18] S.R. Wadia, A Study of $U(N)$ Lattice Gauge Theory in 2-dimensions, arXiv:1212.2906 [INSPIRE].

[19] S.R. Wadia, $N=$ Infinity Phase Transition in a Class of Exactly Soluble Model Lattice Gauge Theories, Phys. Lett. 93B (1980) 403 [INSPIRE]. 
[20] R. Hagedorn, Statistical thermodynamics of strong interactions at high-energies, Nuovo Cim. Suppl. 3 (1965) 147 [INSPIRE].

[21] B. Sundborg, Strings hot fast and heavy, Institute of Theoretical Physics, (1988), https://gupea.ub.gu.se/handle/2077/14375.

[22] L. Susskind, Some speculations about black hole entropy in string theory, hep-th/9309145 [INSPIRE].

[23] G.T. Horowitz and J. Polchinski, A correspondence principle for black holes and strings, Phys. Rev. D 55 (1997) 6189 [hep-th/9612146] [INSPIRE].

[24] M. Hanada, J. Maltz and L. Susskind, Deconfinement transition as black hole formation by the condensation of QCD strings, Phys. Rev. D 90 (2014) 105019 [arXiv:1405.1732] [INSPIRE].

[25] Y. Hidaka and R.D. Pisarski, Zero Point Energy of Renormalized Wilson Loops, Phys. Rev. D 80 (2009) 074504 [arXiv:0907.4609] [INSPIRE].

[26] S. Gupta, K. Huebner and O. Kaczmarek, Renormalized Polyakov loops in many representations, Phys. Rev. D 77 (2008) 034503 [arXiv:0711.2251] [InSPIRE].

[27] A. Mykkanen, M. Panero and K. Rummukainen, Casimir scaling and renormalization of Polyakov loops in large-N gauge theories, JHEP 05 (2012) 069 [arXiv:1202.2762] [INSPIRE].

[28] A. Dumitru, J. Lenaghan and R.D. Pisarski, Deconfinement in matrix models about the Gross-Witten point, Phys. Rev. D 71 (2005) 074004 [hep-ph/0410294] [INSPIRE].

[29] H. Nishimura, R.D. Pisarski and V.V. Skokov, Finite-temperature phase transitions of third and higher order in gauge theories at large N, Phys. Rev. D 97 (2018) 036014 [arXiv: 1712.04465] [INSPIRE].

[30] B. de Wit, J. Hoppe and H. Nicolai, On the Quantum Mechanics of Supermembranes, Nucl. Phys. B 305 (1988) 545 [inSPIRE].

[31] E. Witten, Bound states of strings and p-branes, Nucl. Phys. B 460 (1996) 335 [hep-th/9510135] [INSPIRE].

[32] T. Banks, W. Fischler, S.H. Shenker and L. Susskind, $M$ theory as a matrix model: A conjecture, Phys. Rev. D 55 (1997) 5112 [hep-th/9610043] [INSPIRE].

[33] N. Itzhaki, J.M. Maldacena, J. Sonnenschein and S. Yankielowicz, Supergravity and the large $N$ limit of theories with sixteen supercharges, Phys. Rev. D 58 (1998) 046004 [hep-th/9802042] [INSPIRE].

[34] N. Kawahara, J. Nishimura and S. Takeuchi, Phase structure of matrix quantum mechanics at finite temperature, JHEP 10 (2007) 097 [arXiv: 0706.3517] [INSPIRE].

[35] D.E. Berenstein, J.M. Maldacena and H.S. Nastase, Strings in flat space and pp waves from $N=4$ superYang-Mills, JHEP 04 (2002) 013 [hep-th/0202021] [INSPIRE].

[36] M.S. Costa, L. Greenspan, J. Penedones and J. Santos, Thermodynamics of the BMN matrix model at strong coupling, JHEP 03 (2015) 069 [arXiv:1411.5541] [INSPIRE].

[37] J. Maldacena and A. Milekhin, To gauge or not to gauge?, JHEP 04 (2018) 084 [arXiv: 1802.00428] [INSPIRE].

[38] E. Berkowitz, E. Rinaldi, M. Hanada, G. Ishiki, S. Shimasaki and P. Vranas, Precision lattice test of the gauge/gravity duality at large- $N$, Phys. Rev. D 94 (2016) 094501 [arXiv: 1606.04951] [INSPIRE]. 
[39] E. Berkowitz, M. Hanada, E. Rinaldi and P. Vranas, Gauged And Ungauged: A Nonperturbative Test, JHEP 06 (2018) 124 [arXiv: 1802.02985] [INSPIRE].

[40] O. Aharony, J. Marsano, S. Minwalla and T. Wiseman, Black hole-black string phase transitions in thermal 1+1 dimensional supersymmetric Yang-Mills theory on a circle, Class. Quant. Grav. 21 (2004) 5169 [hep-th/0406210] [INSPIRE].

[41] S. Catterall, A. Joseph and T. Wiseman, Thermal phases of D1-branes on a circle from lattice super Yang-Mills, JHEP 12 (2010) 022 [arXiv:1008.4964] [INSPIRE].

[42] S. Catterall, R.G. Jha, D. Schaich and T. Wiseman, Testing holography using lattice super-Yang-Mills theory on a 2-torus, Phys. Rev. D 97 (2018) 086020 [arXiv:1709.07025] [INSPIRE].

[43] O.J.C. Dias, J.E. Santos and B. Way, Localised and nonuniform thermal states of super-Yang-Mills on a circle, JHEP 06 (2017) 029 [arXiv:1702.07718] [INSPIRE].

[44] T. Harmark, Small black holes on cylinders, Phys. Rev. D 69 (2004) 104015 [hep-th/0310259] [INSPIRE].

[45] R. Gregory and R. Laflamme, Black strings and p-branes are unstable, Phys. Rev. Lett. 70 (1993) 2837 [hep-th/9301052] [INSPIRE].

[46] Y. Aoki, G. Endrodi, Z. Fodor, S.D. Katz and K.K. Szabo, The order of the quantum chromodynamics transition predicted by the standard model of particle physics, Nature $\mathbf{4 4 3}$ (2006) 675 [hep-lat/0611014] [INSPIRE].

[47] S. Muroya, A. Nakamura, C. Nonaka and T. Takaishi, Lattice QCD at finite density: An introductory review, Prog. Theor. Phys. 110 (2003) 615 [hep-lat/0306031] [InSPIRE].

[48] N. Jokela, A. Pönni and A. Vuorinen, Small black holes in global AdS spacetime, Phys. Rev. D 93 (2016) 086004 [arXiv: 1508.00859] [INSPIRE].

[49] O.J. Dias, J.E. Santos and B. Way, Localised $A d S_{5} \times S^{5}$ Black Holes, Phys. Rev. Lett. 117 (2016) 151101 [arXiv: 1605.04911] [INSPIRE].

[50] L.G. Yaffe, Large $N$ phase transitions and the fate of small Schwarzschild-AdS black holes, Phys. Rev. D 97 (2018) 026010 [arXiv:1710.06455] [INSPIRE].

[51] D. Marolf, Microcanonical Path Integrals and the Holography of small Black Hole Interiors, JHEP 09 (2018) 114 [arXiv: 1808.00394] [INSPIRE].

[52] D. Schaich, Progress and prospects of lattice supersymmetry, in 36th International Symposium on Lattice Field Theory (Lattice 2018) East Lansing, MI, United States, July 22-28, 2018, 2018, arXiv:1810.09282 [INSPIRE].

[53] M. Hanada, J. Nishimura, Y. Sekino and T. Yoneya, Direct test of the gauge-gravity correspondence for Matrix theory correlation functions, JHEP 12 (2011) 020 [arXiv: 1108.5153] [INSPIRE].

[54] D. Berenstein, Negative specific heat from non-planar interactions and small black holes in $A d S / C F T$, arXiv:1810.07267 [INSPIRE].

[55] D. Sumpter, Soccermatics: mathematical adventures in the beautiful game, Bloomsbury Publishing, (2016).

[56] O. Aharony, O. Bergman, D.L. Jafferis and J. Maldacena, $N=6$ superconformal Chern-Simons-matter theories, M2-branes and their gravity duals, JHEP 10 (2008) 091 [arXiv: 0806.1218] [INSPIRE].

[57] V. Pestun et al., Localization techniques in quantum field theories, J. Phys. A 50 (2017) 440301 [arXiv: 1608.02952] [INSPIRE]. 